\title{
Thurston norm and cosmetic surgeries
}

\author{
Yi NI \\ Department of Mathematics, Caltech, MC 253-37 \\ 1200 E California Blvd, Pasadena, CA 91125 \\ Email: yni@caltech.edu
}

\begin{abstract}
Two Dehn surgeries on a knot are called cosmetic if they yield homeomorphic manifolds. For a null-homologous knot with certain conditions on the Thurston norm of the ambient manifold, if the knot admits cosmetic surgeries, then the surgery coefficients are equal up to sign.
\end{abstract}

\section{Introduction}

Heegaard Floer homology is a powerful theory introduced by Ozsváth and Szabó 9 . One important aspect of Heegaard Floer homology is that it behaves well under Dehn surgeries. In fact, if one knows about the knot Floer complex of a knot, then one can compute the Heegaard Floer homology of any surgery on the knot 11, 14, 13. This makes Heegaard Floer homology very useful in the study of Dehn surgery.

In this paper, we will use Heegaard Floer homology to study cosmetic surgeries. We first recall the definition of cosmetic surgeries.

Definition 1.1. If two Dehn surgeries on a knot yield homeomorphic manifolds, then these two surgeries are cosmetic.

Cosmetic surgeries are very rare. More precisely, one has the following Cosmetic Surgery Conjecture.

Conjecture 1.2. [5, Problem 1.81] Suppose $K$ is a knot in a closed manifold $Y$. If the complement of $K$ is irreducible and is not the solid torus, then any two surgeries on $K$ do not yield manifolds which are homeomorphic via an orientation preserving homeomorphism.

The main theorem of this paper is an analogue of [13, Theorem 9.7] and [8, Theorem 1.5]. See also [16].

All manifolds in this paper are oriented, unless otherwise stated.

Theorem 1.3. Suppose $Y$ is a closed 3-manifold with $b_{1}(Y)>0$. Let $K$ be a null-homologous knot in $Y$, then the inclusion map $Y-K \rightarrow Y$ induces an isomorphism $H_{2}(Y-K) \cong H_{2}(Y)$, so we can identify $H_{2}(Y)$ with $H_{2}(Y-K)$. 
Suppose $r \in \mathbb{Q} \cup\{\infty\}$, let $Y_{r}(K)$ be the manifold obtained by $r$-surgery on $K$. Suppose $(Y, K)$ satisfies that

$$
x_{Y}(h)<x_{Y-K}(h), \quad \text { for any nonzero element } h \in H_{2}(Y) .
$$

Here $x_{M}$ is the Thurston norm [15] in $M$. The conclusion is, if two rational numbers $r, s$ satisfy that $Y_{r}(K) \cong \pm Y_{s}(K)$, then $r= \pm s$.

Sometimes the condition (11) can be weakened if there is a certain additional condition. For example, we can prove the following theorem.

Theorem 1.4. Suppose $Y$ is a closed 3-manifold with $b_{1}(Y)>0$. Suppose $K$ is a null-homologous knot in $Y$. Suppose $x_{Y} \equiv 0$, while the restriction of $x_{Y-K}$ on $\mathrm{H}_{2}(Y)$ is nonzero. Then we have the same conclusion as Theorem 1.3. Namely, if two rational numbers $r, s$ satisfy that $Y_{r}(K) \cong \pm Y_{s}(K)$, then $r= \pm s$.

Acknowledgements. The author is partially supported by an AIM Five-Year Fellowship and NSF grant number DMS-0805807.

\section{Non-triviality theorems}

In this section, we will state some non-triviality theorems in Heegaard Floer homology. We first set up some notations we will use in this paper.

Let $Y$ be a closed 3-manifold. Suppose $\mathfrak{S}$ is a subset of $\operatorname{Spin}^{c}(Y)$, let

$$
H F^{\circ}(Y, \mathfrak{S})=\bigoplus_{\mathfrak{s} \in \mathfrak{S}} H F^{\circ}(Y, \mathfrak{s})
$$

where $H F^{\circ}$ is one of $\widehat{H F}, H F^{\infty}, H F^{+}, H F^{-}$. Furthermore, if $h \in H_{2}(Y)$, then

$$
H F^{\circ}(Y, h, i)=\bigoplus_{\mathfrak{s} \in \operatorname{Spin}^{c}(Y),\left\langle c_{1}(\mathfrak{s}), h\right\rangle=2 i} H F^{\circ}(Y, \mathfrak{s}) .
$$

Similarly, if $F$ is a Seifert surface for a knot $K \subset Y$, then

$$
\widehat{H F K}(Y, K,[F], i)=\bigoplus_{\xi \in \underline{\operatorname{Spin}^{c}}(Y, K),\left\langle c_{1}(\xi), \widehat{F}\right\rangle=2 i} \widehat{\operatorname{HFK}}(Y, K, \xi),
$$

see [1] for more details. Following Kronheimer and Mrowka [7, let

$$
H F^{\circ}(Y \mid h)=H F^{\circ}\left(Y, h, \frac{1}{2} x(h)\right) .
$$

A very important feature of Heegaard Floer homology is that it detects the Thurston norm of a 3-manifold. In [10, this result is stated for universally twisted Heegaard Floer homology. Nevertheless, this result should also hold if one uses untwisted coefficients. In fact, the analogous result for Monopole Floer homology is stated with untwisted coefficients [6, Corollary 41.4.2]. In order to state our results, we first recall two definitions. 
Definition 2.1. Suppose $M$ is a compact 3-manifold, a properly embedded surface $S \subset M$ is taut if $x(S)=x([S])$ in $H_{2}(M, \partial S)$, no proper subsurface of $S$ is null-homologous, and if any component of $S$ lies in a homology class that is represented by an embedded sphere then this component is a sphere. Here $x(\cdot)$ is the Thurston norm.

Definition 2.2. Suppose $K$ is a null-homologous knot in a closed 3-manifold $Y$. An oriented surface $F \subset Y$ is a Seifert-like surface for $K$, if $\partial F=K$. When $F$ is connected, we say that $F$ is a Seifert surface for $K$. We also view a Seifert-like surface as a proper surface in $Y-\stackrel{\circ}{\nu}(K)$.

As in the proof of [2, Theorem 2.2], using the known non-triviality results for twisted coefficients stated in [8] and the Universal Coefficients Theorem, we can prove the following theorems. (The same results can also be proved via the approach taken in [4, 7].)

Theorem 2.3. Suppose $Y$ is a closed 3-manifold, $h \in H_{2}(Y)$, then

$$
H F^{+}(Y \mid h) \otimes \mathbb{Q} \neq 0, \quad \widehat{H F}(Y \mid h) \otimes \mathbb{Q} \neq 0 .
$$

Theorem 2.4. Suppose $K$ is a null-homologous knot in a closed 3-manifold $Y$. Let $F$ be a taut Seifert-like surface for $K$. Then

$$
\widehat{H F K}\left(Y, K,[F], \frac{x(F)+1}{2}\right) \otimes \mathbb{Q} \neq 0 .
$$

\section{A surgery formula}

Suppose $K \subset Y$ is a null-homologous knot. Let $Y_{p / q}(K)$ denote the manifold obtained by $\frac{p}{q}$-surgery on $K$. Note that there is a natural identification

$$
\operatorname{Spin}^{c}\left(Y_{p / q}(K)\right) \cong \operatorname{Spin}^{c}(Y) \times \mathbb{Z} / p \mathbb{Z}
$$

Let $\pi: \operatorname{Spin}^{c}\left(Y_{p / q}(K)\right) \rightarrow \operatorname{Spin}^{c}(Y)$ be the projection to the first factor.

The goal of this section is to prove the following theorem, which is a (much easier) analogue of [13, Theorem 1.1].

Theorem 3.1. Suppose $K \subset Y$ is a null-homologous knot. If $\widehat{H F}(Y, \mathfrak{s})=0$, then there exists a constant $C=C(Y, K, \mathfrak{s})$, such that

$$
\operatorname{rank} \widehat{H F}\left(Y_{p / q}(K), \pi^{-1}(\mathfrak{s})\right)=q C .
$$

\subsection{Large surgeries on rationally null-homologous knots}

Suppose $K \subset Y$ is a rationally null-homologous knot. We construct a Heegaard diagram $(\Sigma, \boldsymbol{\alpha}, \boldsymbol{\beta}, w, z)$ for $(Y, K)$, such that $\beta_{1}=\mu$ is a meridian of $K$. Moreover, $w, z$ are two base points associated with a marked point on $\beta_{1}$ as in [11. There is a curve $\lambda \subset \Sigma$ which gives rise to the knot $K$. Doing oriented 
cut-and-pastes to $\lambda$ and $m$ parallel copies of $\mu$, we get a connected simple closed curve supported in a small neighborhood of $\mu \cup \lambda$. We often denote this curve by $m \mu+\lambda$. The $m$ parallel copies of $\mu$ are supported in a small neighborhood of $\mu$. We call this neighborhood the winding region for $m \mu+\lambda . \quad(\Sigma, \boldsymbol{\alpha}, \boldsymbol{\gamma}, z)$ is a diagram for $Y_{m \mu+\lambda}(K)$, where $\gamma_{1}=m \mu+\lambda$ and all other $\gamma_{i}$ 's are small Hamiltonian translations of $\beta_{i}$ 's.

Definition 3.2. As in [13, Section 4], one defines a map

$$
\Xi: \operatorname{Spin}^{c}\left(Y_{m \mu+\lambda}(K)\right) \rightarrow \underline{\operatorname{Spin}^{c}}(Y, K)
$$

as follows. If $\mathfrak{t} \in \operatorname{Spin}^{c}\left(Y_{m \mu+\lambda}(K)\right)$ is represented by a point $\mathbf{y}$ supported in the winding region, let $\mathbf{x} \in \mathbb{T}_{\alpha} \cap \mathbb{T}_{\beta}$ be the "nearest point", and let $\psi \in \pi_{2}(\mathbf{y}, \Theta, \mathbf{x})$ be a small triangle. Then

$$
\Xi(\mathfrak{t})=\underline{\mathfrak{s}}_{w, z}(\mathbf{x})+\left(n_{w}(\psi)-n_{z}(\psi)\right) \cdot \mu .
$$

When we construct the Heegaard triple diagram

$$
(\Sigma, \boldsymbol{\alpha}, \boldsymbol{\beta}, \boldsymbol{\gamma}, w, z)
$$

the position of the meridian $\beta_{1}$ relative to the points in $\lambda \cap \gamma_{1}$ may vary. Our next lemma says that the choice of the position of $\beta_{1}$ does not affect the definition of $\Xi$.

Lemma 3.3. Suppose we have two Heegaard triple diagrams as above

$$
\Gamma_{1}=\left(\Sigma, \boldsymbol{\alpha}, \boldsymbol{\beta}^{1}, \boldsymbol{\gamma}, w^{1}, z^{1}\right), \quad \Gamma_{2}=\left(\Sigma, \boldsymbol{\alpha}, \boldsymbol{\beta}^{2}, \boldsymbol{\gamma}, w^{2}, z^{2}\right)
$$

The two sets $\boldsymbol{\beta}^{1}$ and $\boldsymbol{\beta}^{2}$ differ at the meridian, where the meridian $\beta_{1}^{2} \in \boldsymbol{\beta}^{2}$ is a parallel translation of the meridian $\beta_{1}^{1} \in \boldsymbol{\beta}^{1}$, still supported in the winding region. The two base points are moved together with the meridian.

Using these two diagrams, we can define two maps

$$
\Xi^{1}, \Xi^{2}: \operatorname{Spin}^{c}\left(Y_{m \mu+\lambda}(K)\right) \rightarrow \underline{\operatorname{Spin}^{c}(Y, K)}
$$

Then $\Xi^{1}=\Xi^{2}$.

Proof. Without loss of generality, we may assume there is only one intersection point of $\lambda \cap \gamma_{1}$ between $\beta_{1}^{1}$ and $\beta_{1}^{2}$. See Figure 1 for an illustration.

Suppose $\mathbf{y}^{1}, \mathbf{y}^{2} \in \mathbb{T}_{\alpha} \cap \mathbb{T}_{\gamma}$ are two intersection points supported in the winding region, and suppose their $\gamma_{1}$-coordinates are $y^{1}, y^{2}$, respectively. Assume $\mathfrak{s}_{w^{1}}\left(\mathbf{y}^{1}\right)=\mathfrak{s}_{w^{2}}\left(\mathbf{y}^{2}\right)=\mathfrak{t}$, we want to prove that $\Xi^{1}(\mathfrak{t})=\Xi^{2}(\mathfrak{t})$.

By [9, Lemma 2.19],

$$
\begin{aligned}
& \mathfrak{s}_{w^{1}}\left(\mathbf{y}^{1}\right)-\mathfrak{s}_{w^{1}}\left(\mathbf{y}^{2}\right)=\operatorname{PD}\left(\varepsilon\left(\mathbf{y}^{2}, \mathbf{y}^{1}\right)\right) \\
& \mathfrak{s}_{w^{2}}\left(\mathbf{y}^{2}\right)-\mathfrak{s}_{w^{1}}\left(\mathbf{y}^{2}\right)=\operatorname{PD}(\mu) .
\end{aligned}
$$

Hence $\varepsilon\left(\mathbf{y}^{2}, \mathbf{y}^{1}\right)=\mu$. Let $\tilde{\mathbf{y}}^{1} \in \mathbb{T}_{\alpha} \cap \mathbb{T}_{\gamma}$ be the point whose coordinates coincide with the coordinates of $\mathbf{y}^{1}$, except that its $\gamma_{1}$-coordinate is the next intersection 


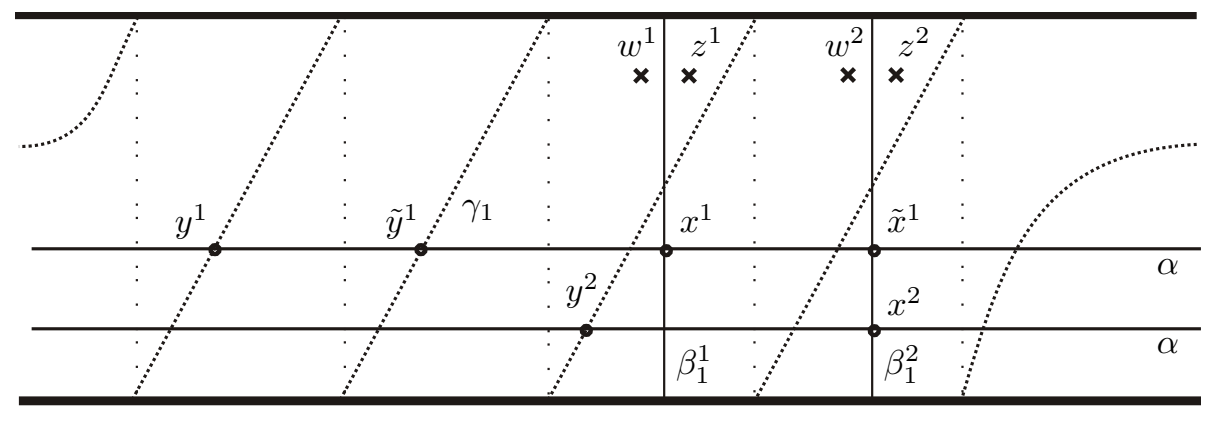

Figure 1: Local picture of the two triple Heegaard diagrams

point to $y^{1}$ on the same $\alpha$-curve, denoted $\tilde{y}^{1}$. Then $\varepsilon\left(\tilde{\mathbf{y}}^{1}, \mathbf{y}^{1}\right)=\mu$, so $\tilde{\mathbf{y}}^{1}$ is in the same equivalence class as $\mathbf{y}^{2}$.

Now we only need to prove that

$$
\Xi^{1}\left(\mathfrak{s}_{w^{1}}\left(\mathbf{y}^{1}\right)\right)=\Xi^{2}\left(\mathfrak{s}_{w^{2}}\left(\tilde{\mathbf{y}}^{1}\right)\right) .
$$

Let $\mathbf{x}^{1} \in \mathbb{T}_{\alpha} \cap \mathbb{T}_{\beta^{1}}, \tilde{\mathbf{x}}^{1} \in \mathbb{T}_{\alpha} \cap \mathbb{T}_{\beta^{2}}$ be the nearest points to $\mathbf{y}^{1}, \tilde{\mathbf{y}}^{1}$, respectively. It is clear that $\underline{\mathfrak{s}}_{w^{1}, z^{1}}\left(\mathbf{x}^{1}\right)=\underline{\mathfrak{s}}_{w^{2}, z^{2}}\left(\tilde{\mathbf{x}}^{1}\right)$. Moreover, the small triangle for $\tilde{\mathbf{y}}^{1}$ in $\Gamma_{2}$ is just a translation of the small triangle for $\mathbf{y}^{1}$ in $\Gamma_{1}$, so they contribute the same $n_{w}(\psi)-n_{z}(\psi)$ term in (2). So (3) follows.

Remark 3.4. In [13, in order to define $\Xi(\mathfrak{t})$, one places the meridian in a position such that the equivalence class of intersection points representing $\mathfrak{t}$ is supported in the winding region. The above lemma removes this restriction.

Lemma 3.5. Suppose $\xi \in \operatorname{Spin}^{c}(Y, K)$. For all sufficiently large $m$, there exists $\mathfrak{t} \in \operatorname{Spin}^{c}\left(Y_{m \mu+\lambda}(K)\right)$, such that $\Xi(\mathfrak{t})=\xi$.

Proof. Let $\mathfrak{s} \in \operatorname{Spin}^{c}(Y)$ be the underlying $\operatorname{Spin}^{c}$ structure of $\xi$. We can choose a Heegaard diagram for $(Y, K)$ such that some $\mathbf{x} \in \mathbb{T}_{\alpha} \cap \mathbb{T}_{\beta}$ represents $\mathfrak{s}$, then $\xi=\underline{\mathfrak{s}}_{w, z}(\mathbf{x})+n \cdot \mu$ for some $n \in \mathbb{Z}$. Now our desired result follows from the definition of $\Xi$.

The following proposition is a part of [13, Theorem 4.1].

Proposition 3.6. Let $K \subset Y$ be a rationally null-homologous knot in a closed, oriented three-manifold, equipped with a framing $\lambda$. Let

$$
\widehat{A}_{\xi}(Y, K)=C_{\xi}\{\max \{i, j\}=0\},
$$

where $C_{\xi}=C F K^{\infty}(Y, K, \xi)$ as in [13]. Then, for all sufficiently large $m$ and all $\mathfrak{t} \in \operatorname{Spin}^{c}\left(Y_{m \mu+\lambda}(K)\right)$, there is an isomorphism

$$
\Psi_{\mathfrak{t}, m}: \widehat{C F}\left(Y_{m \mu+\lambda}(K), \mathfrak{t}\right) \rightarrow \widehat{A}_{\Xi(\mathfrak{t})}(Y, K) .
$$




\subsection{Rational surgeries on null-homologous knots}

Let $K$ be a null-homologous knot in $Y$. As in [13, Section 7], $Y_{\frac{p}{q}}(K)$ can be realized by a Morse surgery with coefficient $a$ on the knot $K^{\prime}=K \# O_{q / r} \subset$ $Y^{\prime}=Y \# L(q, r)$, where $O_{q / r}$ is a $U$-knot in $L(q, r), p=a q+r$. Let

$$
\Xi^{\prime}: \operatorname{Spin}^{c}\left(Y_{a \mu^{\prime}+\lambda^{\prime}}^{\prime}\right) \rightarrow \underline{\operatorname{Spin}^{c}}\left(Y^{\prime}, K^{\prime}\right)
$$

be the map defined in Definition 3.2
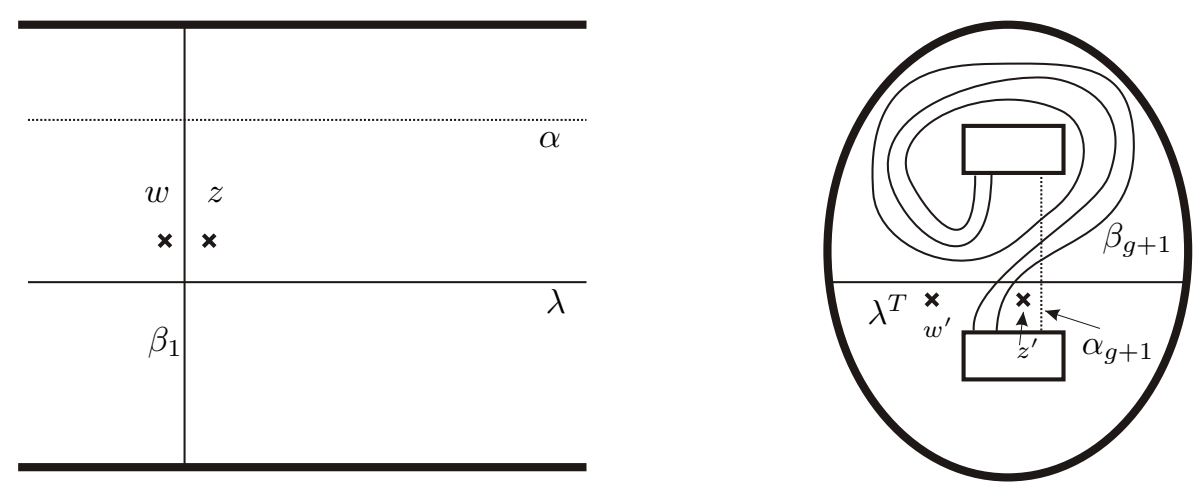

Figure 2: The left hand side is a piece of a Heegaard diagram for $(Y, K)$. The right hand side is a genus 1 Heegaard diagram for $\left(L(q, r), O_{q / r}\right)$. The boundary of the oval is capped off with a disk, and the boundaries of the two rectangles are glued together via a reflection. Here we choose $q=3, r=2$.

\section{Construction 3.7. Let}

$$
\left(\Sigma, \boldsymbol{\alpha}=\left\{\alpha_{1}, \ldots, \alpha_{g}\right\}, \boldsymbol{\beta}=\left\{\beta_{1}, \ldots, \beta_{g}\right\}, w, z\right)
$$

be a doubly-pointed Heegaard diagram for $(Y, K)$, such that $\beta_{1}$ is a meridian for $K$ and the two base points are induced from a marked point on $\beta_{1}$. Suppose $\lambda \subset \Sigma$ represents a longitude of $K$.

Let

$$
\left(T,\left\{\alpha_{g+1}\right\},\left\{\beta_{g+1}\right\}, w^{\prime}, z^{\prime}\right)
$$

be a genus 1 Heegaard diagram for $\left(L(q, r), O_{q / r}\right)$. As in Figure 2, $\beta_{g+1}$ intersects $\alpha_{g+1}$ exactly $q$ times and intersects the boundary of each rectangle exactly $r$ times. Suppose $\lambda^{T} \subset T$ represents a longitude of $O_{q / r}$.

We perform the connected sum of $\Sigma$ and $T$ by identifying the neighborhoods of $z$ and $w^{\prime}$, hence we get a new genus $(g+1)$ surface $\Sigma^{\prime}$. Then

$$
\left(\Sigma^{\prime}, \boldsymbol{\alpha}^{\prime}=\boldsymbol{\alpha} \cup\left\{\alpha_{g+1}\right\}, \boldsymbol{\beta}^{\prime}=\boldsymbol{\beta} \cup\left\{\beta_{g+1}\right\}, w, z^{\prime}\right)
$$

is a Heegaard diagram for $\left(Y^{\prime}, K^{\prime}\right)$. The longitude $\lambda^{\prime}$ of $K^{\prime}$ is a connected sum of $\lambda$ and $\lambda^{T}$. 
We define

$$
\Pi_{1}: \underline{\operatorname{Spin}^{c}}\left(Y^{\prime}, K^{\prime}\right) \rightarrow \underline{\operatorname{Spin}^{c}(Y, K)}
$$

as follows. Given $\xi^{\prime} \in \operatorname{Spin}^{c}\left(Y^{\prime}, K^{\prime}\right)$, suppose $\mathbf{x}^{\prime} \in \mathbb{T}_{\alpha^{\prime}} \cap \mathbb{T}_{\beta^{\prime}}$ represents the underlying $\operatorname{Spin}^{c}$ structure of $\xi^{\prime}$, then

$$
\xi^{\prime}=\underline{\mathfrak{s}}_{w, z^{\prime}}\left(\mathbf{x}^{\prime}\right)+n \cdot \mu^{\prime}
$$

for some $n \in \mathbb{Z}$. Now let $\mathbf{x}$ be the projection of $\mathbf{x}^{\prime}$ to $\mathbb{T}_{\alpha} \cap \mathbb{T}_{\beta}$, then

$$
\Pi_{1}\left(\xi^{\prime}\right)=\underline{\mathfrak{s}}_{w, z}(\mathbf{x})+n \cdot \mu
$$

The following proposition is obvious. (See also [13, Corollary 5.3].)

Proposition 3.8. For any $\xi^{\prime} \in \operatorname{Spin}^{c}\left(Y^{\prime}, K^{\prime}\right)$, we have

$$
C F K^{\infty}\left(Y^{\prime}, K^{\prime}, \xi^{\prime}\right) \cong C F K^{\infty}\left(Y, K, \Pi_{1}\left(\xi^{\prime}\right)\right)
$$

as $\mathbb{Z} \oplus \mathbb{Z}$-filtered chain complexes.

Lemma 3.9. When $m$ is sufficiently large, we have

$$
\pi=G_{Y, K} \circ \Pi_{1} \circ \Xi^{\prime}
$$

Here $G_{Y, K}: \underline{\operatorname{Spin}^{c}}(Y, K) \rightarrow \operatorname{Spin}^{c}(Y)$ is the map defined in [13, Section 2.2].

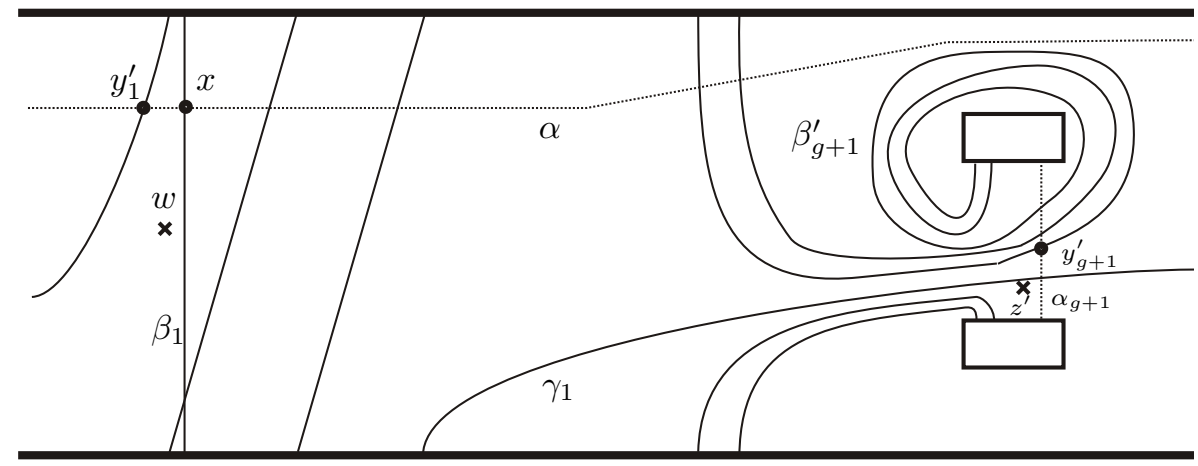

Figure 3: A Heegaard diagram for $Y_{a \mu^{\prime}+\lambda^{\prime}}^{\prime}\left(K^{\prime}\right)$. Here we choose $a=3$.

Proof. We follow the notation in Construction 3.7. Since $\lambda^{\prime}$ intersects $\beta_{1}$ exactly once, we can slide $\beta_{g+1}$ over $\beta_{1} r$ times to eliminate the intersection points in $\beta_{g+1} \cap \lambda^{\prime}$. The new curve is denoted $\beta_{g+1}^{\prime}$ as in Figure 3 . Then

$$
\left(\Sigma^{\prime}, \boldsymbol{\alpha}^{\prime}, \boldsymbol{\beta}^{\prime \prime}=\boldsymbol{\beta} \cup\left\{\beta_{g+1}^{\prime}\right\}, w, z^{\prime}\right)
$$




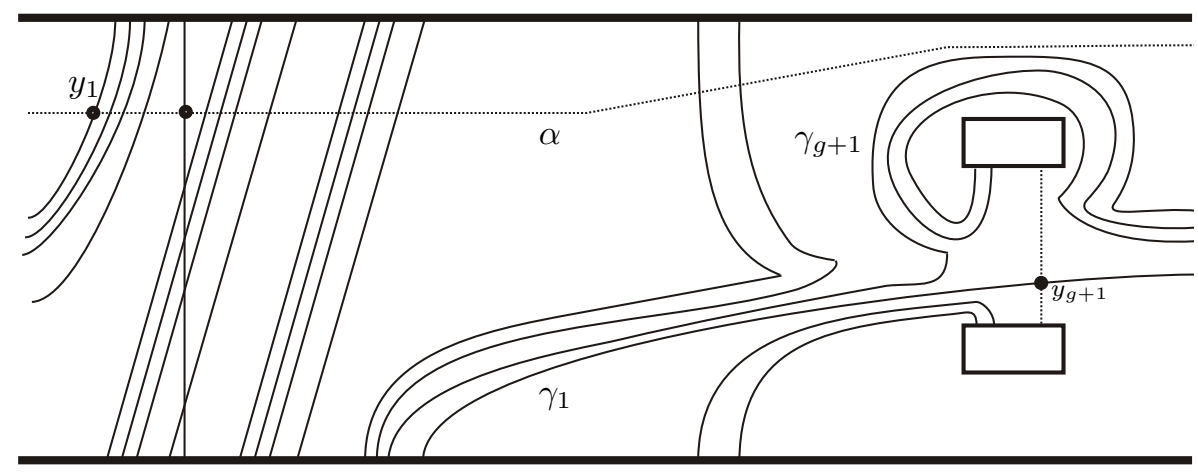

Figure 4: After $q$ handleslides, we get a Heegaard diagram for $Y_{p / q}(K)$.

is also a Heegaard diagram for $\left(Y^{\prime}, K^{\prime}\right)$. Let $\gamma_{1}=a \beta_{1}+\lambda^{\prime}$, then

$$
\left(\Sigma^{\prime}, \boldsymbol{\alpha}^{\prime}, \boldsymbol{\gamma}_{1}=\left\{\gamma_{1}, \beta_{2}, \ldots, \beta_{g}, \beta_{g+1}^{\prime}\right\}, w\right)
$$

is a Heegaard diagram for $Y_{a \mu^{\prime}+\lambda^{\prime}}^{\prime}\left(K^{\prime}\right)$.

The curve $\alpha_{g+1}$ intersects $\gamma_{1}$ exactly once. We can slide $\beta_{g+1}^{\prime}$ over $\gamma_{1} q$ times to eliminate its $q$ intersection points with $\alpha_{g+1}$. The new curve is denoted $\gamma_{g+1}$ as in Figure 4 Now

$$
\left(\Sigma^{\prime}, \boldsymbol{\alpha}^{\prime}, \gamma_{2}=\left\{\gamma_{1}, \beta_{2}, \ldots, \beta_{g}, \gamma_{g+1}\right\}, w\right)
$$

is a Heegaard diagram for $Y_{a \mu^{\prime}+\lambda^{\prime}}^{\prime}\left(K^{\prime}\right)=Y_{p / q}(K)$. Moreover, we may slide other $\alpha$-curves over $\alpha_{g+1}$ to eliminate their intersection points with $\gamma_{1}$. A destabilization will remove $\alpha_{g+1}$ and $\gamma_{1}$. Now we get a diagram

$$
\left(\Sigma^{*}, \boldsymbol{\alpha}^{*}, \boldsymbol{\gamma}^{*}, w\right)
$$

which is isomorphic to

$$
\left(\Sigma, \boldsymbol{\alpha},\left\{\beta_{2}, \ldots, \beta_{g}, \gamma_{g+1}^{*}\right\}, w\right),
$$

where $\gamma_{g+1}^{*}$ is the image of $\gamma_{g+1}$ under the destabilization.

We want to show that $\gamma_{g+1}^{*}$ is isotopic to $p \mu+q \lambda$, the curve obtained by doing cut-and-pastes to $p$ parallel copies of $\mu$ and $q$ parallel copies of $\lambda$. In fact, $\gamma_{g+1}^{*}$ is supported in a small neighborhood of $\mu \cup \lambda$, so it must be isotopic to $p^{\prime} \mu+q^{\prime} \lambda$ for some $p^{\prime}, q^{\prime}$. It is easy to compute the intersection numbers of $\gamma_{g+1}$ with $\lambda$ and $\mu=\beta_{1}$, which are $p=a q+r$ and $q$. The intersection numbers of $\gamma_{g+1}^{*}$ with $\mu$ and $\lambda$ remains the same, so $\gamma_{g+1}^{*}=p \mu+q \lambda$.

Suppose $\mathfrak{t} \in \operatorname{Spin}^{c}\left(Y_{p / q}(K)\right)$. We want to prove

$$
\pi(\mathfrak{t})=G_{Y, K} \circ \Pi_{1} \circ \Xi^{\prime}(\mathfrak{t}) .
$$


We first consider the right hand side of (44). Let $\mathbf{y}^{\prime}$ be a point in $\mathbb{T}_{\alpha^{\prime}} \cap \mathbb{T}_{\gamma_{1}}$ which is supported in the winding region and represents $\mathfrak{t}$ (Figure 3). Suppose the $\gamma_{1}$-coordinate of $\mathbf{y}^{\prime}$ is $y_{1}^{\prime}$ and the $\beta_{g+1}^{\prime}$-coordinate is $y_{g+1}^{\prime}$.

Let $\mathbf{x}^{\prime} \in \mathbb{T}_{\alpha^{\prime}} \cap \mathbb{T}_{\beta^{\prime \prime}}$ be the nearest point to $\mathbf{y}^{\prime}$, then (2) implies that

$$
\Xi^{\prime}(\mathfrak{t})=\underline{\mathfrak{s}}_{w, z^{\prime}}\left(\mathbf{x}^{\prime}\right)+n \cdot \mu^{\prime}
$$

for some $n \in \mathbb{Z}$. Let $\mathbf{x}$ be the projection of $\mathbf{x}^{\prime}$ to $\mathbb{T}_{\alpha} \cap \mathbb{T}_{\beta}$, then

$$
\Pi_{1} \circ \Xi^{\prime}(\mathfrak{t})=\underline{\mathfrak{s}}_{w, z}(\mathbf{x})+n \cdot \mu .
$$

Hence

$$
G_{Y, K} \circ \Pi_{1} \circ \Xi^{\prime}(\mathfrak{t})=\mathfrak{s}_{w}(\mathbf{x}) .
$$

Now we consider the left hand side of (4). As in Figure 4, we get another Heegaard diagram for $Y_{p / q}(K)$ by $q$ handle slides. In this diagram, we can find a point $\mathbf{y} \in \mathbb{T}_{\alpha^{\prime}} \cap \mathbb{T}_{\gamma_{2}}$ which represents $\mathfrak{t}$ as $\mathbf{y}^{\prime}$ does. In fact, since $\alpha_{g+1}$ intersects $\gamma_{1}$ exactly once and is disjoint from other $\gamma$-curves, $\mathbf{y}$ must contain the intersection point of $\alpha_{g+1}$ and $\gamma_{1}$, denoted $y_{g+1}$. The $\gamma_{1}$-coordinate of $\mathbf{y}$, called $y_{1}$, is determined by $y_{1}^{\prime}$ and $y_{g+1}^{\prime}$ : it is one of the $q$ intersection points on $\gamma_{g+1}$ near $y_{1}^{\prime}$, and the choice among these $q$ points is specified by the position of $y_{g+1}^{\prime}$. Other coordinates of $\mathbf{y}$ are the same as $\mathbf{y}^{\prime}$.

After handleslides and one destabilization, we get a point $\mathbf{y}^{*} \in \mathbb{T}_{\alpha^{*}} \cap \mathbb{T}_{\gamma^{*}}$ whose coordinates are the same as $\mathbf{x}$ except that its $\gamma_{1}$-coordinate is $y_{1}$. So its nearest point in $\mathbb{T}_{\alpha} \cap \mathbb{T}_{\beta}$ is $\mathbf{x}$, hence $\mathbf{x}$ represents $\pi(\mathfrak{t})$. This proves (4).

Lemma 3.10. Let $H\left(\widehat{A}_{\xi}(Y, K)\right)$ be the homology of the chain complex $\widehat{A}_{\xi}(Y, K)$. For a fixed $\xi$, when $|n| \gg 0$,

$$
H\left(\widehat{A}_{\xi+n \cdot \mu}(Y, K)\right) \cong \widehat{H F}\left(Y, G_{Y, K}(\xi)\right) .
$$

Proof. By the definitions

$$
\begin{aligned}
\widehat{A}_{\xi+n \cdot \mu}(Y, K) & =C_{\xi+n \cdot \mu}\{\max \{i, j\}=0\} \\
& =C_{\xi}\{\max \{i, j-n\}=0\} .
\end{aligned}
$$

By the adjunction inequality, $H\left(C_{\xi}\{i, j\}\right)=0$ when $|i-j| \gg 0$. So

$$
H\left(C_{\xi}\{\max \{i, j-n\}=0\}\right) \cong H\left(C_{\xi}\{i=0\}\right)
$$

when $n \gg 0$. The latter group is isomorphic to $\widehat{H F}\left(Y, G_{Y, K}(\xi)\right)$ by [13, Proposition 3.2].

When $n \ll 0$, we have

$$
H\left(C_{\xi}\{\max \{i, j-n\}=0\}\right) \cong H\left(C_{\xi}\{j=n\}\right) \cong H\left(C_{\xi}\{j=0\}\right),
$$

which is isomorphic to $\widehat{H F}\left(Y, G_{Y,-K}(\xi)\right)$ by [13, Proposition 3.2]. Now by [13. Equation (4)] and the fact that $K$ is null-homologous, we have $G_{Y, K}(\xi)=$ $G_{Y,-K}(\xi)$. 
Lemma 3.11. Suppose $\widehat{H F}(Y, \mathfrak{s})=0$, then $H\left(\widehat{A}_{\xi^{\prime}}\left(Y^{\prime}, K^{\prime}\right)\right) \neq 0$ for only finitely many $\xi^{\prime} \in\left(G_{Y, K} \circ \Pi_{1}\right)^{-1}(\mathfrak{s})$.

Proof. For each $\xi \in \operatorname{Spin}^{c}(Y, K)$, there are exactly $q$ relative $\operatorname{Spin}^{c}$ structures in $\Pi_{1}^{-1}(\xi)$. Moreover, by Proposition 3.8 , if $\xi^{\prime} \in \Pi_{1}^{-1}(\xi)$, then

$$
\widehat{A}_{\xi^{\prime}}\left(Y^{\prime}, K^{\prime}\right) \cong \widehat{A}_{\xi}(Y, K) \text {. }
$$

Hence we only need to show that $H\left(\widehat{A}_{\xi}(Y, K)\right) \neq 0$ for only finitely many $\xi \in G_{Y, K}^{-1}(\mathfrak{s})$.

Pick any $\xi \in G_{Y, K}^{-1}(\mathfrak{s})$, then

$$
G_{Y, K}^{-1}(\mathfrak{s})=\{\xi+i \cdot \mu \mid i \in \mathbb{Z}\} .
$$

By Lemma 3.10, $H\left(\widehat{A}_{\xi+i \cdot \mu}(Y, K)\right)$ is isomorphic to $\widehat{H F}(Y, \mathfrak{s})$ when $|i|$ is large, hence is 0 . This finishes the proof.

Proposition 3.12. When $m$ is sufficiently large,

$$
\begin{aligned}
\widehat{H F}\left(Y_{m \mu^{\prime}+\lambda^{\prime}}^{\prime}\left(K^{\prime}\right), \pi^{-1}(\mathfrak{s})\right) & \cong \bigoplus_{\left\{\xi^{\prime} \mid G_{Y, K} \circ \Pi_{1}\left(\xi^{\prime}\right)=\mathfrak{s}\right\}} H\left(\widehat{A}_{\xi^{\prime}}\left(Y^{\prime}, K^{\prime}\right)\right) \\
& \cong \bigoplus_{\left\{\xi \mid G_{Y, K}(\xi)=\mathfrak{s}\right\}}^{q} H\left(\widehat{A}_{\xi}(Y, K)\right) .
\end{aligned}
$$

Proof. By Proposition 3.6, when $m$ is sufficiently large

$$
\widehat{H F}\left(Y_{m \mu^{\prime}+\lambda^{\prime}}^{\prime}\left(K^{\prime}\right), \pi^{-1}(\mathfrak{s})\right) \cong \bigoplus_{\mathfrak{t} \in \pi^{-1}(\mathfrak{s})} H\left(\widehat{A}_{\Xi^{\prime}(\mathfrak{t})}\left(Y^{\prime}, K^{\prime}\right)\right) .
$$

By Lemma 3.9 .

$$
\Xi^{\prime}\left(\pi^{-1}(\mathfrak{s})\right)=\Xi^{\prime}\left(\Xi^{\prime-1} \circ\left(G_{Y, K} \circ \Pi_{1}\right)^{-1}(\mathfrak{s})\right) \subset\left(G_{Y, K} \circ \Pi_{1}\right)^{-1}(\mathfrak{s}) .
$$

Consider the map

$$
\Xi_{\mathfrak{s}}^{\prime}: \pi^{-1}(\mathfrak{s}) \rightarrow\left(G_{Y, K} \circ \Pi_{1}\right)^{-1}(\mathfrak{s}) .
$$

By [8, Lemma 2.4], $\Xi_{\mathfrak{s}}^{\prime}$ is injective. Moreover, by Lemmas 3.5] and 3.11] when $m$ is sufficiently large, the range of $\Xi_{\mathfrak{s}}^{\prime}$ contains all $\xi^{\prime} \in\left(G_{Y, K} \circ \Pi_{1}\right)^{-1}(\mathfrak{s})$ satisfying $H\left(\widehat{A}_{\xi^{\prime}}\left(Y^{\prime}, K^{\prime}\right)\right) \neq 0$. This proves the first equality.

In order to prove the second equality, we note that for each $\xi \in \operatorname{Spin}^{c}(Y, K)$, there are exactly $q$ relative $\operatorname{Spin}^{c}$ structures in $\Pi_{1}^{-1}(\xi)$. Moreover, by Proposition 3.8, if $\xi^{\prime} \in \Pi_{1}^{-1}(\xi)$, then

$$
\widehat{A}_{\xi^{\prime}}\left(Y^{\prime}, K^{\prime}\right) \cong \widehat{A}_{\xi}(Y, K)
$$

So the second equality easily follows. 
Proof of Theorem [3.1, Let

$$
C=\operatorname{rank} \bigoplus_{\left\{\xi \mid G_{Y, K}(\xi)=\mathfrak{s}\right\}} H\left(\widehat{A}_{\xi}(Y, K)\right) .
$$

By Proposition 3.12 ,

$$
\operatorname{rank} \widehat{H F}\left(Y_{p / q}, \pi^{-1}(\mathfrak{s})\right)=q C
$$

when $p$ is sufficiently large.

Since $\widehat{H F}(Y, \mathfrak{s})=0$, we have $\widehat{H F}\left(Y^{\prime}, \mathfrak{s}^{\prime}\right)=0$ for any $\mathfrak{s}^{\prime}$ that extends $\mathfrak{s}$. By [10, Theorem 9.12], we have the long exact sequence

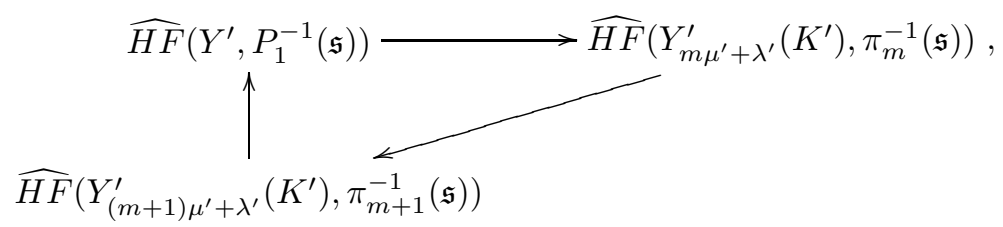

where

$$
\begin{gathered}
P_{1}: \operatorname{Spin}^{c}\left(Y^{\prime}\right) \rightarrow \operatorname{Spin}^{c}(Y), \\
\pi_{m}: \operatorname{Spin}^{c}\left(Y_{m \mu^{\prime}+\lambda^{\prime}}^{\prime}\left(K^{\prime}\right)\right) \rightarrow \operatorname{Spin}^{c}(Y)
\end{gathered}
$$

are the natural projection maps. Since $\widehat{H F}\left(Y^{\prime}, P_{1}^{-1}(\mathfrak{s})\right)=0$, we have

$$
\widehat{H F}\left(Y_{a \mu^{\prime}+\lambda^{\prime}}^{\prime}\left(K^{\prime}\right), \pi_{a}^{-1}(\mathfrak{s})\right) \cong \widehat{H F}\left(Y_{m \mu^{\prime}+\lambda^{\prime}}^{\prime}\left(K^{\prime}\right), \pi_{m}^{-1}(\mathfrak{s})\right)
$$

for $m$ sufficiently large. Hence its rank is always $q C$.

\section{Cosmetic surgeries}

Proof of Theorem 1.3. Assume there are two rational numbers $\frac{p_{1}}{q_{1}}, \frac{p_{2}}{q_{2}}$ satisfying that there is a homeomorphism

$$
f: Y \frac{p_{1}}{q_{1}} \rightarrow \pm Y \frac{p_{2}}{q_{2}}
$$

then $\left|p_{1}\right|=\left|p_{2}\right|$ for homological reasons. If $\frac{p_{1}}{q_{1}} \neq \pm \frac{p_{2}}{q_{2}}$, then we can assume

$$
0<q_{1}<q_{2} \text {. }
$$

Without loss of generality, we may assume $Y-K$ is irreducible. By (1) and the adjunction inequality, we conclude that $\widehat{H F}\left(Y, h, \frac{1}{2} x_{Y-K}(h)\right)=0$. It then follows from Theorem 3.1 that there is a constant $C_{h}$, such that

$$
\operatorname{rank} \widehat{H F}\left(Y_{p / q}(K), h, \frac{1}{2} x_{Y-K}(h)\right)=q C_{h} .
$$


Since (11) holds, [1, Corollary 2.4] implies that

$$
x_{Y-K}(h)=x_{Y_{p / q}(K)}(h)
$$

for any nonzero $h \in H_{2}(Y)$ and $\frac{p}{q} \in \mathbb{Q}$. Theorem 2.3 then implies that

$$
\operatorname{rank} \widehat{H F}\left(Y_{p / q}(K) \mid h\right)=q C_{h} \neq 0 .
$$

Since $K$ is null-homologous, the inclusion maps $Y-K \rightarrow Y_{r}$ induce isomorphisms on $H_{2}$ for each $r \in \mathbb{Q} \cup\{\infty\} \backslash\{0\}$. Hence we can identify $H_{2}\left(Y_{r}(K)\right)$ with $H_{2}(Y)$. Now $f_{*}: H_{2}\left(Y_{\frac{p_{1}}{q_{1}}}\right) \rightarrow H_{2}\left(Y_{\frac{p_{2}}{q_{2}}}\right)$ can be regarded as a map

$$
f_{*}: H_{2}(Y) \rightarrow H_{2}(Y)
$$

Fix a nonzero $h \in H_{2}(Y)$, we have

$$
\operatorname{rank} \widehat{H F}\left(Y_{\frac{p_{1}}{q_{1}}} \mid f_{*}^{n}(h)\right)=\frac{q_{1}}{q_{2}} \operatorname{rank} \widehat{H F}\left(Y_{\frac{p_{2}}{q_{2}}} \mid f_{*}^{n}(h)\right) \neq 0
$$

for any $n \in \mathbb{Z}$. Moreover, since $f: Y_{\frac{p_{1}}{q_{1}}} \rightarrow \pm Y_{\frac{p_{2}}{q_{2}}}$ is a homeomorphism, we have

$$
\operatorname{rank} \widehat{H F}\left(Y_{\frac{p_{1}}{q_{1}}} \mid f_{*}^{n-1}(h)\right)=\operatorname{rank} \widehat{H F}\left(Y_{\frac{p_{2}}{q_{2}}} \mid f_{*}^{n}(h)\right) \text {. }
$$

Thus we get

$$
\operatorname{rank} \widehat{H F}\left(Y_{\frac{p_{1}}{q_{1}}} \mid f_{*}^{n}(h)\right)=\left(\frac{q_{1}}{q_{2}}\right)^{n} \operatorname{rank} \widehat{H F}\left(Y_{\frac{p_{1}}{q_{1}}} \mid h\right) \neq 0 .
$$

So $0<\operatorname{rank} \widehat{H F}\left(Y_{\frac{p_{1}}{q_{1}}} \mid h\right)<1$ when $n$ is sufficiently large, which is impossible.

Proof of Theorem 1.4. Since $x_{Y} \equiv 0$, the adjunction inequality implies that $\widehat{H F}\left(Y, h, \frac{1}{2} x_{Y-K}(h)\right)=0$ for any $h \in H_{2}(Y)$ satisfying $x_{Y-K}(h) \neq 0$. Using Theorems 3.1, 2.3 and [1, Corollary 2.4], we have

$$
\operatorname{rank} \widehat{H F}\left(Y_{p / q}(K) \mid h\right)=q C_{h}
$$

for some nonzero constant $C_{h}$. Now the argument is the same as in the proof of Theorem 1.3 .

\section{References}

[1] D. Gabai, Foliations and the topology of 3-manifolds II, J. Differential Geom. 26 (1987) no. 3, 461-478.

[2] M. Hedden, Y. Ni, Manifolds with small Heegaard Floer ranks, preprint (2009), available at arXiv:0906.4771. 
[3] S. Jabuka, T. Mark, Product formulae for Ozsváth-Szabó 4-manifold invariants, Geom. Topol. 12 (2008) 1557-1651 (electronic).

[4] A. Juhász, Floer homology and surface decompositions, Geom. Topol. 12 (2008), 299-350 (electronic)

[5] R. Kirby, Problems in low-dimensional topology, Geometric topology, Proceedings of the 1993 Georgia International Topology Conference held at the University of Georgia, Athens, GA, August 2-13, 1993. Edited by William H. Kazez. AMS/IP Studies in Advanced Mathematics, 2.2. American Mathematical Society, Providence, RI; International Press, Cambridge, MA, 1997.

[6] P. Kronheimer, T. Mrowka, Monopoles and three-manifolds, New Mathematical Monographs 10, Cambridge University Press, Cambridge (2007).

[7] P. Kronheimer, T. Mrowka, Knots, sutures and excision, preprint (2008), available at arXiv:0807.4891.

[8] Y. Ni, Non-separating spheres and twisted Heegaard Floer homology, preprint (2009), available at arXiv:0902.4034

[9] P. Ozsváth, Z. Szabó, Holomorphic disks and topological invariants for closed three-manifolds, Ann. of Math. (2), 159 (2004), no. 3, 1027-1158.

[10] P. Ozsváth, Z. Szabó, Holomorphic disks and three-manifold invariants: properties and applications, Ann. of Math. (2), 159 (2004), no. 3, 1159-1245.

[11] P. Ozsváth, Z. Szabó, Holomorphic disks and knot invariants, Adv. Math. 186 (2004), no. 1, 58-116.

[12] P. Ozsváth, Z. Szabó, Holomorphic disks and genus bounds, Geom. Topol. 8 (2004), 311-334 (electronic).

[13] P. Ozsváth, Z. Szabó, Knot Floer homology and rational surgeries, preprint (2005), available at arXiv:math.GT/0504404

[14] J. Rasmussen, Floer homology and knot complements, PhD Thesis, Harvard University (2003), available at arXiv:math.GT/0306378

[15] W. Thurston, A norm for the homology of 3-manifolds, Mem. Amer. Math. Soc. 59 (1986), no. 339, i-vi and 99-130.

[16] Z. Wu, Cosmetic Surgery in Integral Homology L-Spaces, preprint (2009), available at arXiv:0911.5333 